\title{
Erratum: Rainbow biotech—South Africa's emerging sector
}

Sabine Louët

Nat. Biotechnol. 24, 1313-1316 (2006); published online 2 November 2006.

In the version of the article initially published, on page 1316, paragraph 4, line 17, the interest rate of the eGoliBio incubator is erroneous. Instead of $3 \%$, the rate is actually prime rate less $3 \%$, which is $\sim 10 \%$. The text should read "They also have access to Rand 100,000 (\$12,600) loans at prime less $3 \%$ interest-rate...."

\section{Corrigendum: Reversal of mouse hepatic failure using an implanted liver-assist device containing ES cell-derived hepatocytes}

Alejandro Soto-Gutiérrez, Naoya Kobayashi, Jorge David Rivas-Carrillo, Nalu Navarro-Álvarez, Debaio Zhao, Teru Okitsu, Hirofumi Noguchi, Hesham Basma, Yashuhiko Tabata, Yong Chen, Kimiaki Tanaka, Michiki Narushima, Atsushi Miki, Tadayoshi Ueda, Hee-Sook Jun, Ji-Won Yoon, Jane Lebkowski, Noriaki Tanaka \& Ira J Fox

Nat. Biotechnol. 24, 1412-1419 (2006); published online 5 November 2006; corrected after print 7 February 2007.

In the version of the article initially published, the fifth author's name is misspelled. The correct spelling is Debiao Zhao. The error has been corrected in the HTML and PDF versions of the article.

\section{Corrigendum: Expression of artificial microRNAs in transgenic} Arabidopsis thaliana confers virus resistance

Qi-Wen Niu, Shih-Shun Lin, Jose Luis Reyes, Kuan-Chun Chen, Hui-Wen Wu, Shyi-Dong Yeh \& Nam-Hai Chua

Nat. Biotechnol. 24, 1420-1428 (2006); published online 22 October 2006; corrected after print 7 February 2007.

In the version of the article initially published, in the Author Contributions, the initials of the last author responsible for the virus challenge and related experiments are H.-W.W. and not H.-W.N., as originally indicated. In Figure 2e, the amiR-P69159 line is line number 1 and not line number 11 , as originally indicated. The error has been corrected in the PDF version of the article. 